\title{
Comparison of Two Equivalent Dipole Layer Based Inverse Electrocardiography Techniques for the Non-Invasive Estimation of His-Purkinje Mediated Ventricular Activation
}

\author{
Machteld J Boonstra ${ }^{1}$, Rob W Roudijk ${ }^{1}$, Peter Loh $^{1}$, Peter M van Dam ${ }^{1}$ \\ ${ }^{1}$ Department of Cardiology, Division Heart \& Lungs, University Medical Center Utrecht, Utrecht \\ University, Utrecht, the Netherlands
}

\begin{abstract}
Non-invasive estimation of the cardiac activation sequence through inverse electrocardiography (iECG) based on the equivalent double layer becomes increasingly difficult for multiple activation waves through the myocardium. Earlier studies simulated the effect of the His-Purkinje system through a multi-focal search, which does not completely take into account the effect of multiple near simultaneous activation waves initiated by the HisPurkinje system. In this study, the iECG initial estimation of the cardiac activation sequence was modified to include electro-anatomical structures associated with the HisPurkinje system to provide a physiologically robust initial estimate. The performance of two iECG techniques for the estimation of different His-Purkinje mediated ventricular activation sequences were tested, i.e. normal activation and left and right bundle branch block activation.
\end{abstract}

\section{Introduction}

Inverse electrocardiography ( $i \mathrm{ECG}$ ) is used to noninvasively estimate the cardiac activation sequence. HisPurkinje mediated ventricular activation generates a complex cardiac activation pattern, resulting in multiple activation waves initiated from multiple endocardial breakthroughs spreading and colliding through the ventricular myocardium $[1,2]$. The ECG waveform is the result of the spatial summation of all these waves, and thus partial cancelation and amplification occurs. Studies of the His-Purkinje system have shown the involvement of electro-anatomical structures like papillary muscles and moderator band with early ventricular activation. Individual anatomy, bundle branch blocks and myocardial disease can alter this His-Purkinje mediated cardiac activation [3].
Cardiac activation sequences can be non-invasively estimated from body surface potential maps (BSPM) in combination with anatomical geometries derived from medical imaging. The non-invasive estimation method used in this study is based on an equivalent double layer (EDL) representing electrical activity at both the endocardium and epicardium [4,5]. In the previously described multi-focal EDL-based $i$ ECG, the existence of multiple near simultaneous foci was appreciated using a multi-focal correlation-based search technique [4]. However, estimating normal ventricular activation requires the incorporation of the effect of the His-Purkinje system on normal activation in the $i$ ECG method to accurately estimate the interplay of multiple simultaneous activation wave fronts. Multi-wave $i$ ECG was designed to mimic this His-Purkinje effect on normal ventricular activation, in contrast to multi-focal $i$ ECG which omits this effect. In this paper, the two $i$ ECG techniques were compared for activation sequences initiated by fully and partially active His-Purkinje systems.

\section{Methods}

\subsection{Materials}

Six subject specific CT-based geometries of the ventricular myocardium, LV and RV blood cavies, thorax and lungs were created. Electro-anatomical structures associated with the His-Purkinje system were incorporated in the ventricular geometries, i.e. two LV papillary muscles and the RV moderator band were segmented in the ventricular geometry (Figure 1). Thorax geometries were registered to 3D images to obtain ECG electrode positions.

Simulation study

Cardiac activation sequences were simulated using the 
fastest route algorithm (FRA) using multiple activation sites from which a resulting activation sequence was computed [3]. Activation patterns of fully (normal) active and partially active His-Purkinje systems were simulated. Normal activation was simulated by merging six activation patterns initiated at all six different His-Purkinje associated endocardial regions. Partially active His-Purkinje systems were simulated as right bundle branch block (RBBB) and left bundle branch block (LBBB) ventricular activation. For RBBB activation, the LV endocardial His-Purkinje associated regions were set active and for LBBB activation, RV endocardial His-Purkinje were set active. Corresponding 67-lead BSPM were simulated using the boundary element method, using conductivity values: 0.2 $\mathrm{S} / \mathrm{m}$ for the thorax and ventricular tissue, $0.04 \mathrm{~S} / \mathrm{m}$ for the lungs and $0.6 \mathrm{~S} / \mathrm{m}$ for all blood cavities. White noise was added to the signals. Simulated QRS complexes were used as input for both $i$ ECG techniques.

\section{Invasive study}

In one subject, five subsequent QRS complexes segmented from measured 67-lead BSPM were used as an input for both $i \mathrm{ECG}$ techniques to estimate the activation sequence using both $i$ ECG techniques. These activation

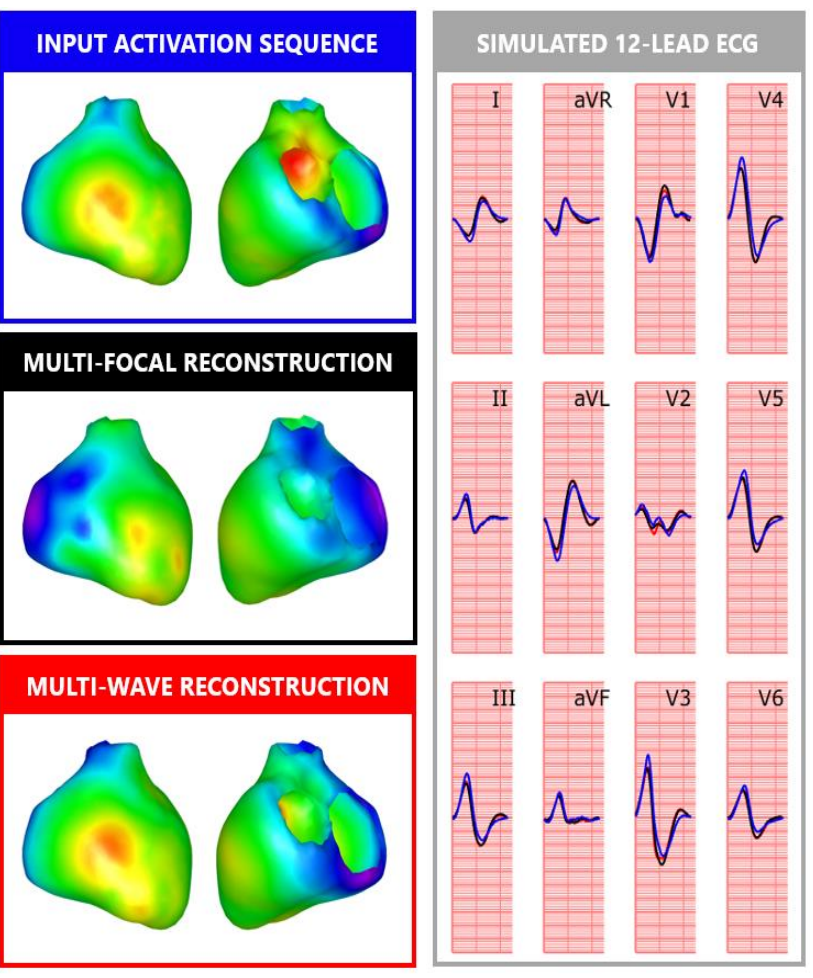

Figure 2: A representative example of the simulation study in a normal ventricular activation sequence. Simulated (left panel, blue) and estimated multi-focal (middle panel, black) and multiwave (lower panel, red) iECG activation sequences had similar 12-lead BSPM (right panel). Inter-map correlation between simulated and multi-focal iECG activation sequence is $63 \%$ and between true and multi-wave iECG activation pattern is $88 \%$.

\section{Cardiac Source Model}

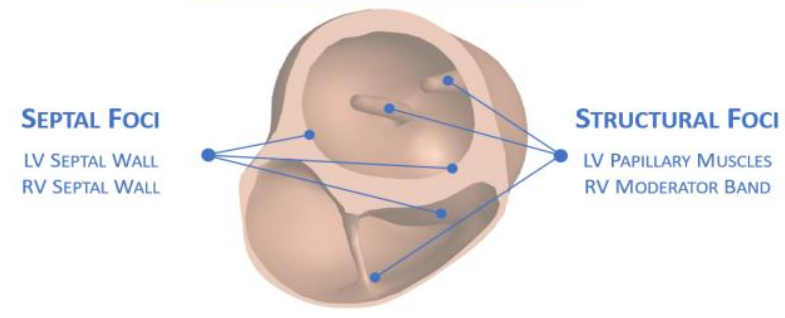

Figure 1: A representation of a subject specific CT-based model with the six regions associated with early ventricular activation and His-Purkinje anatomy [1,2]. Three structural foci: two LV papillary muscles and the moderator band were identified based on cardiac imaging. Three septal regions were automatically identified based on anatomical landmarks in the ventricular model.

sequences were compared to invasive electro-anatomical local activation timing mapping.

\section{2 iECG technique}

Multi-focal $i$ ECG has been described earlier [4]. It serves an iterative approach where the 'fundamental' activation sequence dominates the finally estimated activation sequence. Total activation duration of the 'fundamental' activation sequence was matched to QRS duration by adapting myocardial conduction velocity. Up to five additional foci were iteratively added if the match between simulated and input ECG was improved.

In multi-wave $i E C G$, six His-Purkinje associated regions at the endocardium were identified in the ventricular geometry (Figure 1). Structural foci were identified at the insertion of the of the structure to the ventricular free wall. Septal foci were identified near the $\mathrm{RV}$ endocardial apex, in the lower one third of the LV endocardium and in the upper one third of the antero-septal wall of the LV endocardium. An activation sequence was computed using the FRA with myocardial conduction velocity set at $0.85 \mathrm{~mm} / \mathrm{ms}$. Merging activation sequences initiated at these six regions results in 63 combinations of possible activation sequences. These 63 combinations were tested by correlating simulated BSPM to input BSPM. The combination yielding the highest correlation with measured BSPM was selected as initial estimation of the activation sequence. Initial estimations of both $i$ ECG techniques were further optimized using a dedicated Levenberg-Marquardt procedure with the regularization parameter $\mu^{2}$ set to $5 * 10^{-6} \mathrm{mV}^{2} \mathrm{~ms}^{2} / \mathrm{m}^{2}$

\subsection{Data analysis}

Estimated cardiac activation sequences of both iECG techniques were quantitatively compared to true activation 
based on inter-map correlation and absolute differences between LAT maps, amount of initial foci, myocardial

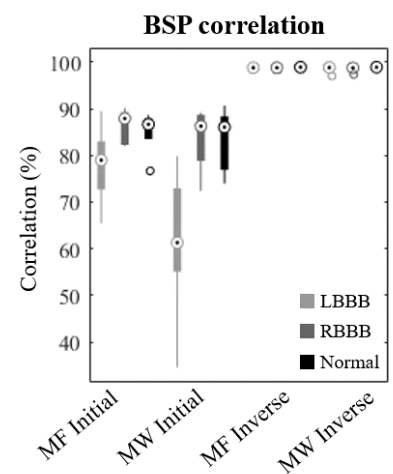

Inter-map correlation and absolute difference

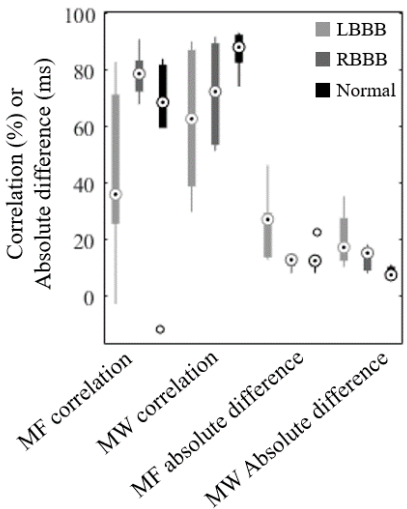

Figure 3: Overview of differences in estimation of the cardiac activation sequence with multi-focal $(M F)$ and multi-wave $(M W)$ iECG technique in six subjects. Normal ventricular activation, left bundle-branch block (LBBB) and right bundle branch block (RBBB) were tested. Median (white dot) and inter-quartile range (thick box) of estimated values in six subjects are displayed per simulated activation pattern. Outliers are displayed using an open dot. Left: inter-BSPM comparison between methods and right: intermap comparison.

conduction velocity and correlation and relative difference between input BSPM and simulated BSPM.

\section{Results}

\section{Simulation study}

In Figure 2, an example of the estimation of normal ventricular activation is displayed. For both estimations BSPM are similar, as reflected in a correlation of $99 \%$ between input BSPM and simulated BSPM. However, the estimated normal activation pattern were different for both techniques, which is reflected in a lower inter-map correlation for multi-focal $i$ ECG compared to multi-wave $i$ ECG. For both $i$ ECG techniques, correlation between input BPS and simulated BSPM was $99 \pm 1 \%$ and relative difference was $16 \pm 3 \%$. As can be observed in Figure 3, correlation between input BSPM and simulated BSPM were comparable for both the initial estimation and the optimized estimation of the cardiac activation sequence using both $i$ ECG techniques.

Mean total activation duration estimated using multifocal $i \mathrm{ECG}$ was $107 \pm 14 \mathrm{~ms}$ for normal ventricular activation, $119 \pm 21 \mathrm{~ms}$ for RBBB ventricular activation and $128 \pm 16 \mathrm{~ms}$ for LBBB ventricular activation. With increasing total activation duration, myocardial conduction velocity decreased from $1.4 \pm 0.3 \mathrm{~mm} / \mathrm{ms}$ for normal ventricular activation, $1.2 \pm 0.3 \mathrm{~mm} / \mathrm{ms}$ for RBBB ventricular activation and $1.1 \pm 0.3$ for $\mathrm{LBBB}$ ventricular activation. For all activation sequences, six foci were identified.

Mean total activation duration estimated using multiwave $i$ ECG was $102 \pm 13 \mathrm{~ms}$ for normal ventricular activation, $125 \pm 18 \mathrm{~ms}$ for RBBB ventricular activation and $150 \pm 18 \mathrm{~ms}$ for LBBB ventricular activation. The number of initial foci decreased with increasing total activation duration: $5.5 \pm 0.5$ for normal activation, $5 \pm 0.9$ for RBBB activation and $3.5 \pm 0.5$ for LBBB activation. Foci were identified at the LV endocardial surface respectively $\mathrm{RV}$ endocardial surface: $3.5 \pm 0.5$ respectively $2.0 \pm 0$ for normal activation, $3.7 \pm 0.5$ respectively $1.3 \pm 0.5$ for RBBB activation and $1.8 \pm 0.4$ respectively $1.7 \pm 0.5$ for $\mathrm{LBBB}$ activation. Larger differences between estimated initial total activation duration and true QRS duration decreased inter-map correlation, even after the optimization procedure.

\section{Invasive study}

In one subject with a narrow QRS complex $(90 \mathrm{~ms})$ correlation between input BSPM and simulated BSPM was $99 \%$ and relative difference was $15 \%$ for both $i \mathrm{ECG}$ techniques. Epicardial inter-map correlatoin was $-4 \pm 8 \%$

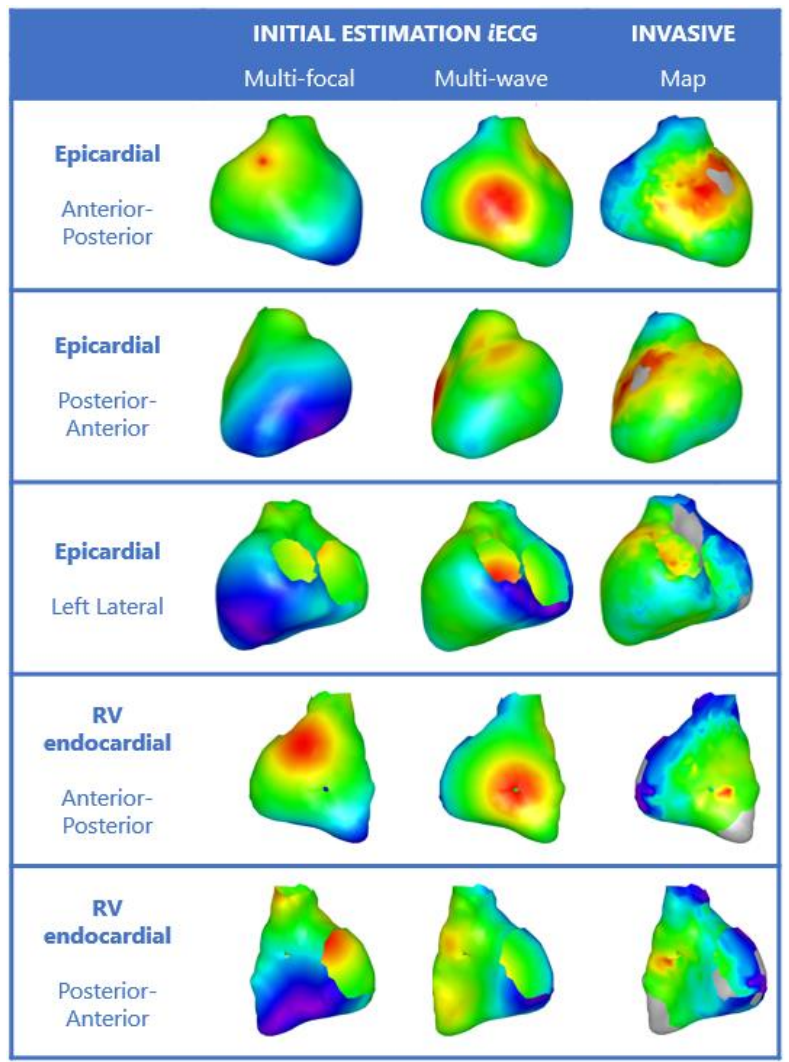

Figure 4: The activation pattern estimated by both iECG procedures compared to invasive mapping. In this case, epicardial inter-map correlation between invasive and estimated activation sequences was $3 \%$ for multi-focal $i E C G$ and $65 \%$ for multi-wave iECG. RV endocardial inter-map correlation was $-10 \%$ for multi-focal iECG and $69 \%$ for multiwave iECG. 
for multi-focal $i \mathrm{ECG}$ and $65 \pm 1 \%$ for multi-wave $i \mathrm{ECG}$. $\mathrm{RV}$ endocardial inter-map correlation was $-5 \pm 7 \%$ for multi-focal $i \mathrm{ECG}$ and $68 \pm 0 \%$ for multi-wave $i \mathrm{ECG}$. An example of an estimated activation sequence using both $i$ ECG algorithms is depicted in Figure 4.

\section{Discussion}

In this paper, the performance of two $i \mathrm{ECG}$ techniques is compared in six subjects through a simulation study and preliminary data of one subject with invasive mapping are presented. In multi-wave $i \mathrm{ECG}$, the effect of the HisPurkinje on ventricular activation was mimicked, whereas in multi-wave $i$ ECG it was omitted. Overall, performance of multi-wave $i$ ECG is equal or better compared to multifocal $i \mathrm{ECG}$, especially in narrow QRS complexes as indicted by the inter-map correlation and absolute difference.

Physiologically, with decreasing QRS duration an increased number of initial sites of activation are present at the endocardial surface [1,3]. With decreasing QRS duration, myocardial conduction velocity was increased by the multi-focal method whereas the number of foci increased in multi-wave $i$ ECG. Subject specific myocardial conduction velocity as determined in multifocal $i$ ECG may be true if one initial site of activation is present, but becomes rather unrealistic in patients with normal QRS complexes where multiple foci reduce total activation duration. Furthermore, multi-focal $i \mathrm{ECG}$ is likely to identify an average of near simultaneous foci as a first focus, resulting in an inadequate estimation. Multifocal $i E C G$ is found to be the most effective in rather monophasic, simple activation patterns like PVCs. However, as it omits the effect of the His-Purkinje system on ventricular activation, the technique is inadequate to be used for normal ventricular activation, which was also indicated by the results as the performance of multi-focal $i$ ECG increased with increasing QRS duration. In contrast, multi-wave $i$ ECG posed a more physiologically realistic estimation of the ventricular activation sequence by incorporating true cardiac physiology. With decreasing QRS duration, the number of foci increased. Multi-wave $i$ ECG takes the interplay of several existing waves into account and is therefore electro-physiologically more realistic.

The optimization procedure is designed to minimize the relative difference between input and recorded BSPM by tuning LAT. The optimization procedure will thus tune the activation sequence, but no foci will appear or disappear as an effect of the procedure. Therefore, a physiologically realistic initial estimation is of utmost importance, thus favoring multi-wave $i$ ECG. In this study, the optimization procedure both positively and negatively affected the intermap correlation and absolute differences. With increasing difference between initially estimated total activation duration and true QRS duration, multi-wave $i$ ECG performed worse. These results emphasize both the need for a physiologically realistic initial estimation and additional regularization of the optimization procedure to cardiac physiology.

The main aim of the simulation study was to assess the capability of both methods to recapture activation sequences with multiple foci. Therefore, anisotropic heart models were used, but incompletely reflecting true cardiac tissue characteristics. Furthermore, in the invasive study, a structurally diseased heart was mapped. The results presented in this paper show the improved performance of multi-wave iECG compared to multi-focal $i \mathrm{ECG}$ in the estimation of narrow QRS complexes, also in the subject who underwent the invasive mapping. However, further validation of multi-wave $i \mathrm{ECG}$ using invasive mapping is required. Also, the effect of inhomogeneities (e.g. scar, fibrofatty tissue formation) in cardiac tissue on multi-wave $i$ ECG will be assessed.

\section{Conclusion}

The results show that non-invasive estimation of normal ventricular activation is better captured by multi-wave $i$ ECG compared to multi-focal $i$ ECG. Multi-wave $i \mathrm{ECG}$ capabilities have to be further investigated with patient data and invasively measured activation timings.

\section{Acknowledgements}

This work was supported by the Netherlands Cardiovascular Research Initiative, an initiative with support of the Dutch Heart Foundation (grant numbers CVON2015-12 eDETECT and QRS-Vision 2018B007).

\section{References}

[1] Durrer D, Van Dam RT, Freud GE, Janse MJ, Meijler FL, Arzbaecher RC. Total excitation of the isolated human heart. Circulation. 1970 Jun;41(6):899-912.

[2] Tawara S. Das Reizleitungenssystem des Säugetierherzens: eine anatomisch-histologische Studie über das Atrioventikularbündel und die Purkinjeschen Fäden. Verslag von Gustav Fischer. 1906.

[3] Hatala R, Savard P, Tremblay G, Page P, Cardinal R, Molin F, Kus T, Nadeau R. Three distinct patterns of ventricular activation in infarcted human hearts. An interoperative cardiac mapping study during sinus rhythm. Circulation 1995 Mar; 1;91(5):1480-94.

[4] van Dam PM, Oostendorp TF, Linnenbank AC, van Oosterom A. Non-invasive imaging of cardiac activation and recovery. Ann Biomed Eng. 2009 Sep;37(9):1739-56.

[5] van Oosterom, A. Genesis of the T wave as based on an equivalent surface source model. Journal of Electrocardiography 2001; 34 (Supplement): 217-227.

Address for correspondence: Machteld Boonstra, The Netherlands, E-mail address: machteldboonstra@gmail.com 